\title{
Efficacy of folic acid supplementation on endothelial function and plasma homocysteine concentration in coronary artery disease: A meta-analysis of randomized controlled trials
}

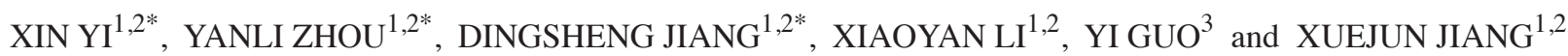 \\ ${ }^{1}$ Department of Cardiology, Renmin Hospital of Wuhan University; ${ }^{2}$ Cardiovascular Research Institute of Wuhan University; \\ ${ }^{3}$ Department of Epidemiology, School of Public Health of Wuhan University, Wuhan, Hubei 430060, P.R. China
}

Received September 25, 2013; Accepted January 28, 2014

DOI: $10.3892 /$ etm.2014.1553

\begin{abstract}
The aim of the present study was to conduct an updated meta-analysis of relevant randomized controlled trials (RCTs) in order to estimate the effect of folic acid supplementation on endothelial function and the concentration of plasma homocysteine in patients with coronary artery disease (CAD). An extensive search of PubMed was conducted to identify RCTs that compared folic acid with placebo therapy. The mean difference (MD) and 95\% confidence interval (CI) were used as a measure of the correlation between folic acid supplementation and endothelial function/plasma homocysteine concentration. Of the 377 patients included in this analysis, 191 patients underwent folic acid supplementation and 186 individuals underwent placebo treatment. Compared with the use of a placebo, folic acid supplementation alone exhibited significant efficacy on increasing flow-mediated dilation (FMD; MD, $57.72 \mu \mathrm{m}$; 95\% CI, 50.14-65.31; $\mathrm{P}<0.05)$ and lowering the concentration of plasma homocysteine (MD, $-3.66 \mu \mathrm{mol} / 1 ; 95 \% \mathrm{CI}$, -5.44--1.87; $\left.\mathrm{P}<0.05 ; \mathrm{I}^{2}, 87 \%\right)$. There was no significant change in the response to end diastolic diameter, glyceryl-trinitrate diameter, heart rate, baseline and peak hyperemic flow and systolic and diastolic blood pressure between the folic acid and placebo groups $(\mathrm{P}>0.05)$. Therefore, the meta-analysis indicated that $5 \mathrm{mg}$ folic acid daily supplementation for $>4$ weeks significantly improved FMD and lowered the concentration of plasma homocysteine in patients with CAD. However, more RCTs are required in order to confirm these observations.
\end{abstract}

Correspondence to: Professor Xuejun Jiang, Department of Cardiology, Renmin Hospital of Wuhan University, 238 Jiefang Road, Wuhan, Hubei 430060, P.R. China

E-mail: xjjiang@whu.edu.cn

*Contributed equally

Key words: folic acid, endothelial function, homocysteine, coronary artery disease, meta-analysis

\section{Introduction}

Endothelial dysfunction is closely associated with the occurrence and development of atherosclerotic disease and numerous studies have confirmed that coronary artery disease (CAD) is often accompanied by endothelial dysfunction $(1,2)$. A number of studies have demonstrated that hyperhomocysteinemia, one of the risk factors of CAD, promotes the occurrence and development of CAD by damaging vascular endothelial function (3-5). Experimental studies (6-8) and epidemiological data (9) have demonstrated that combined folic acid (400 $\mu \mathrm{g}-5 \mathrm{mg}$ daily) and vitamin B therapy may be involved in the regulation of vascular endothelial structure and function. However, there is no definitive conclusion with regard to this effect lowering the concentration of plasma homocysteine. Whether long-term high-dose folic acid (5 mg daily) alone may effectively improve vascular endothelial function and lower the concentration of plasma homocysteine in patients with CAD remains controversial (10-12). Therefore, a meta-analysis of randomized controlled trials (RCTs) with regard to folic acid treatment for CAD was performed to verify whether folic acid is capable of improving endothelial function and reducing the concentration of plasma homocysteine in patients with CAD.

\section{Materials and methods}

Search strategy for RCTs. In order to compare the efficacy of folic acid supplementation or placebos for CAD, an extensive literature search on PubMed was performed in order to locate relevant RCTs that were published between January 1966 and September 2012. A flow chart of the search methodology used in this meta-analysis is provided in Fig. 1. A total of 42,714 reports were identified using a PubMed query of 'folic acid' or 'folate'. Limits of 'endothelial/endothelial function' and 'homocysteine' reduced the number of reports to 475 . Further refinement of the search criteria with the addition of 'coronary artery disease/coronary heart disease' reduced the number of reports to 104. Finally, limits of 'randomized controlled trial/RCT' resulted in a total of 23 reports $(6,13-31)$. The titles and abstracts of the reports were reviewed for terms, including folic acid or folate, endothelial function, CAD 


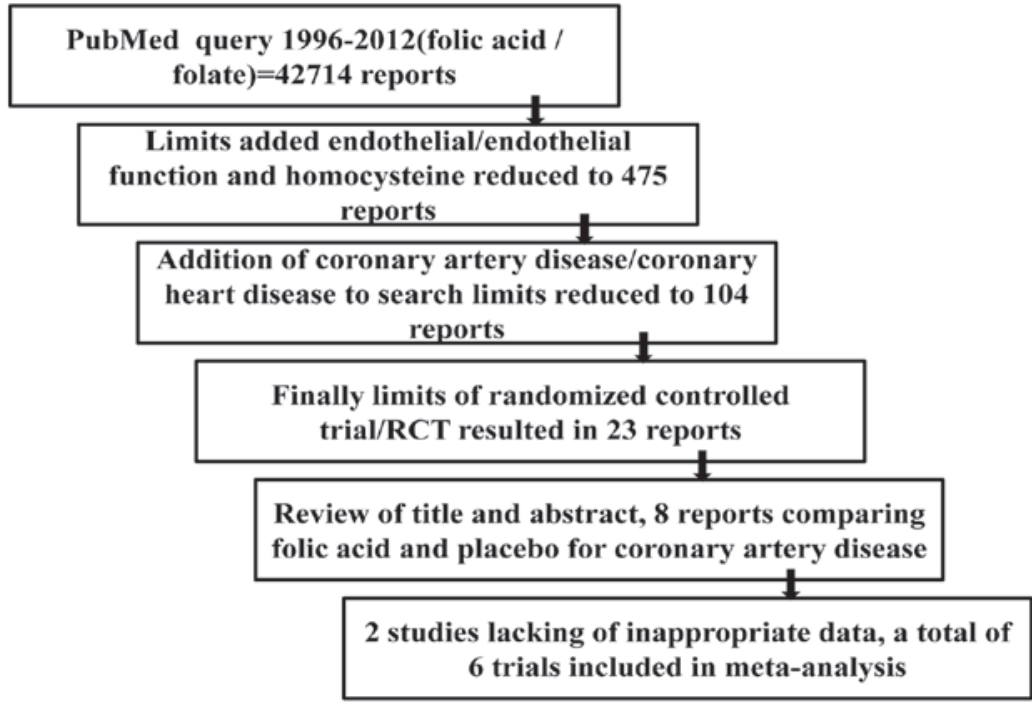

Figure 1. Search process for inclusion in this meta-analysis comparing the efficacy of folic acid supplementation on endothelial function and plasma homocysteine concentration in patients with CAD. CAD, coronary artery disease.

or coronary heart disease. Following careful review, eight randomized studies were identified that discussed folic acid and placebo treatment of CAD. However, two studies $(10,28)$ were excluded for their imprecise information with regard to flow-mediated dilation (FMD) and end diastolic diameter (EDD) in the folic acid and placebo-treated groups. As a result, a total of six trials $(11,12,17,25,27,31)$ were used for this meta-analysis. The studies were reviewed by two independent authors in order to assess their quality. Any discrepancies in their judgments were resolved by joint discussion or discussion with a third reviewer, referencing the original report. Variable trials that were assessed included an accurate description of methods including study design, inclusion criteria, exclusion criteria, the statistical tests used, the baselines between the patients undergoing folic acid or placebo treatment and the outcome of the measures reported along with the results of the follow-up.

Inclusion/exclusion criteria. Studies were eligible for inclusion if they met the following criteria: i) the study was a RCT; ii) the study was conducted using human subjects with CAD; iii) active treatment consisted of folic acid supplementation (without additional vitamin B supplementation); iv) folic acid was administered orally with a dose of $5 \mathrm{mg} /$ day; v) the duration of active treatment was $\geq 4$ weeks and $\leq 16$ weeks; vi) plasma homocysteine concentration was provided; vii) the study reported the mean FMD and/or EDD for the treatment and placebo groups. Studies that reported either FMD or EDD changes alone, assuming all other criteria were met, were included in this meta-analysis.

Although 23 potentially relevant studies were identified and screened, 17 trials did not meet the inclusion criteria for this meta-analysis. Major reasons for the exclusion of a study were i) the patients were also treated with vitamin B; ii) the subject populations did not have CAD; iii) the dosage of folic acid was not $5 \mathrm{mg} /$ day; iv) the trials were not randomized; v) there was an absence of data by which to calculate the changes in FMD or EDD.
Data abstraction and statistical analysis. Information with regard to study design, sample size, duration, clinical characteristics and the medication of the participant, as well as biochemical parameters and treatment results regarding endothelial function, were independently abstracted from the six clinical trials and subsequently entered as standard forms into a Microsoft Excel (Microsoft Corporation, Redmond, Washington, WA, USA) spreadsheet to calculate the overall efficacy following folic acid supplementation compared with that following the administration of a placebo. The risk of bias was assessed as recommended in the Cochrane Handbook by RevMan 5.0 (The Cochrane Collaboration) and the standards of assessment were as follows: i) adequate sequence generation; ii) allocation concealment; iii) incomplete outcome data were addressed; iv) free of selective reporting; v) free of other bias. On the basis of this assessment methodology, the two reviewers provided each eligible study with an overall rating of low, high or an unclear risk of bias. Once the outcomes had been evaluated, a table summarizing the observations was created using the Grading of Recommendations Assessment, Development and Evaluation (GRADE) system.

The data were analyzed according to the intention-to-treat principle. RevMan 5.0 was used for the meta-analysis. The mean difference (MD) with $95 \%$ confidence interval (CI) were calculated as a measure of the correlation between folic acid supplementation and endothelial function/plasma homocysteine concentration. A two-sided $\mathrm{P}<0.05$ was considered to indicate a statistically significant difference.

$\mathrm{P}$ values from $\chi^{2}$ statistical analysis and $\mathrm{I}^{2}$ were used for the heterogeneity test. Heterogeneity was considered to be significant when $\mathrm{P}<0.05$. If $\mathrm{P}>0.1$ or $\mathrm{I}^{2}<50 \%$, 'there may be no heterogeneity among included studies and summarize data across the trials by selecting a fixed-effects model with the software RevMan 5.0' and pooled data across the trials by selecting a fixed-effects model based on inverse variance methods. Otherwise, the results were considered to have "considerable heterogeneity' and were compared using a random-effects model. Publication bias was assessed by funnel plots with the 
standard error of the intervention effect on the vertical axis and MD measuring the effect of intervention on the horizontal axis.

\section{Results}

Characteristics of included RCTs. Participant and study design characteristics for the six RCTs included in the meta-analysis are shown in Table I. Among these clinical studies, four trials had a parallel double-blind design and two had a crossover double-blind design. Of the 377 patients included, 191 patients underwent folic acid supplementation and 186 patients underwent placebo treatment. The majority of trials included aged male participants. Trial duration varied between eight weeks and four months. The medication administered in the variable trials is presented in Table II. There were no differences in the baseline clinical or biochemical parameters (Tables I and II). The majority of patients were treated with antiplatelet therapy, lipid-lowering therapy, $\beta$-blockers, angiotensin-converting-enzyme inhibitors, nitrates and other drug therapies. Biochemical parameters, including total cholesterol, triglycerides, low-density lipoprotein (LDL) and high-denstity lipoprotein cholesterol, plasma folic acid, vitamin $\mathrm{B}_{12}$, creatinine and glucose, are shown in Table III. Furthermore, baseline endothelial function data of the folic acid and placebo groups are presented in Table IV.

Assessment of the bias risk and recommended classification of included studies. The bias risk for the included trials was assessed according to the assessment methodology recommended by The Cochrane Collaboration (Figs. 2 and 3). Adequate sequence generation and allocation concealment were not described clearly in all six studies. The six trials reported complete outcome data, but one study did not clearly describe the selective reporting (12). Due to incomplete information in four studies $(12,25,27,31)$, there may be other biases. The efficacy of folic acid supplementation on FMD and the concentration of plasma homocysteine was the main outcome in this meta-analysis. The recommended classification of FMD was deemed to be of low quality, but the grade of evidence for the concentration of plasma homocysteine was deemed to be of moderate quality. Therefore, due to the quality of evidence according to the GRADE system, the use of folic acid is recommended to reduce the concentration of plasma homocysteine.

Efficacy of folic acid on endothelial function. The individual trial results for the effects of folic acid and placebo therapy on FMD, EDD, glyceryl-trinitrate (GTN) diameter change, heart rate, baseline and peak hyperemic flow, systolic and diastolic blood pressure (BP) and the pooled estimate of the effect are shown in Fig. 4. Of the six trials included, four studies measured the efficacy of folic acid on FMD; the pooled estimate from these studies exhibited a marked increase in FMD in the folic acid-treated group when compared with the placebo group (MD, 57.72 $\mu \mathrm{m} ; 95 \% \mathrm{CI}, 50.14-65.31 ; \mathrm{P}<0.05 ; \mathrm{I}^{2}$, $0 \%$ ). Using a random-effects versus a fixed-effects model did not markedly alter the pooled estimate. However, the pooled estimate presented no significant difference in the response to EDD (MD, -0.03; 95\% CI, -0.20-0.15; P=0.75; I², 0\%), GTN diameter change $\left(\mathrm{MD}, 1.74 ; 95 \% \mathrm{CI},-17.13-20.61 ; \mathrm{P}=0.86 ; \mathrm{I}^{2}\right.$,
$0 \%)$, heart rate $\left(\mathrm{MD},-0.39 ; 95 \% \mathrm{CI},-2.89-2.11 ; \mathrm{P}=0.76 ; \mathrm{I}^{2}\right.$, $0 \%$ ), baseline hyperemic flow (MD, 1.02; 95\% CI, -4.81-6.84; $\mathrm{P}=0.73 ; \mathrm{I}^{2}, 0 \%$ ), peak hyperemic flow (MD, $-2.25 ; 95 \% \mathrm{CI}$, -23.32-18.82; $\left.\mathrm{P}=0.83 ; \mathrm{I}^{2}, 0 \%\right)$, systolic BP (MD, $-1.07 ; 95 \% \mathrm{CI}$, -5.71-3.03; $\left.\mathrm{P}=0.61 ; \mathrm{I}^{2}, 0 \%\right)$ and diastolic $\mathrm{BP}(\mathrm{MD}, 0.08,95 \% \mathrm{CI}$, $-2.10-2.27 ; \mathrm{P}=0.94 ; \mathrm{I}^{2}, 0 \%$ ) between the folic acid and placebo treatment groups when using a fixed-effects model. A funnel plot of effect size versus study precision was asymmetrical with a relative dearth of moderately precise negative studies, indicating the presence of a positive publication bias (Fig. 5).

Effect of folic acid on the concentration of plasma homocysteine. In total, five studies reported a change in the concentration of plasma homocysteine (Fig. 6). The results from the random-effects model pooling the MD demonstrated that folic acid supplementation correlated with a significant reduction in the concentration of plasma homocysteine (MD, $\left.-3.66 \mu \mathrm{mol} / 1 ; 95 \% \mathrm{CI},-5.44--1.87 ; \mathrm{P}<0.05 ; \mathrm{I}^{2}, 87 \%\right)$. Using a fixed-effects versus random-effects model did not substantially alter the pooled estimate.

\section{Discussion}

The results of the present meta-analysis demonstrated that an increase in FMD and decrease in plasma homocysteine concentration in CAD patients were associated with folic acid supplementation. However, there was no significant change in EDD, GTN diameter, heart rate, baseline and peak hyperemic flow and systolic and diastolic BP between the folic acid supplementation and placebo-treated groups. Measuring the FMD of a brachial artery using color Doppler ultrasound may accurately reflect the state of coronary endothelial function and serve as a non-invasive method to evaluate endothelial function, thus, is of great value clinically $(32,33)$. Notably, the results of the current RCT meta-analysis are in agreement with data from prospective cohort studies, indicating the efficacy of high-dose folic acid supplementation in improving endothelial function and lowering the concentration of plasma homocysteine in subjects with CAD.

It has been recognized that hyperhomocysteinemia is a risk factor for CAD. Compared with individuals without CAD, the risk of CAD increases 2-fold in patients with hyperhomocysteinemia (34). The function of folic acid is limited to decreasing the levels of plasma cysteine initially, but subsequent studies have demonstrated that folic acid (400 $\mu \mathrm{g} /$ day) can markedly reduce plasma homocysteine levels, while a larger dose of folic acid improves endothelial function in patients with CAD and reduces the incidence of cardiovascular events $(11,13)$. Doshi et al (25) observed that an improvement in FMD occurred prior to a significant drop in plasma homocysteine concentration with folic acid treatment, indicating that the enhancement was not explained by a reduction in homocysteine levels. Following the administration of folic acid, FMD improved markedly at $2 \mathrm{~h}$ and peaked $4 \mathrm{~h}$ after the first dose. However, there was no significant decrease in the total or free plasma homocysteine levels in the $4 \mathrm{~h}$ following the initial dose of folic acid. Verhaar et al (35) demonstrated that 5-methyltetrahydrofolate, a major circulating folate, is capable of improving endothelial function in patients with familial hypercholesterolemia who are free of vascular disease and are not receiving lipid-lowering 


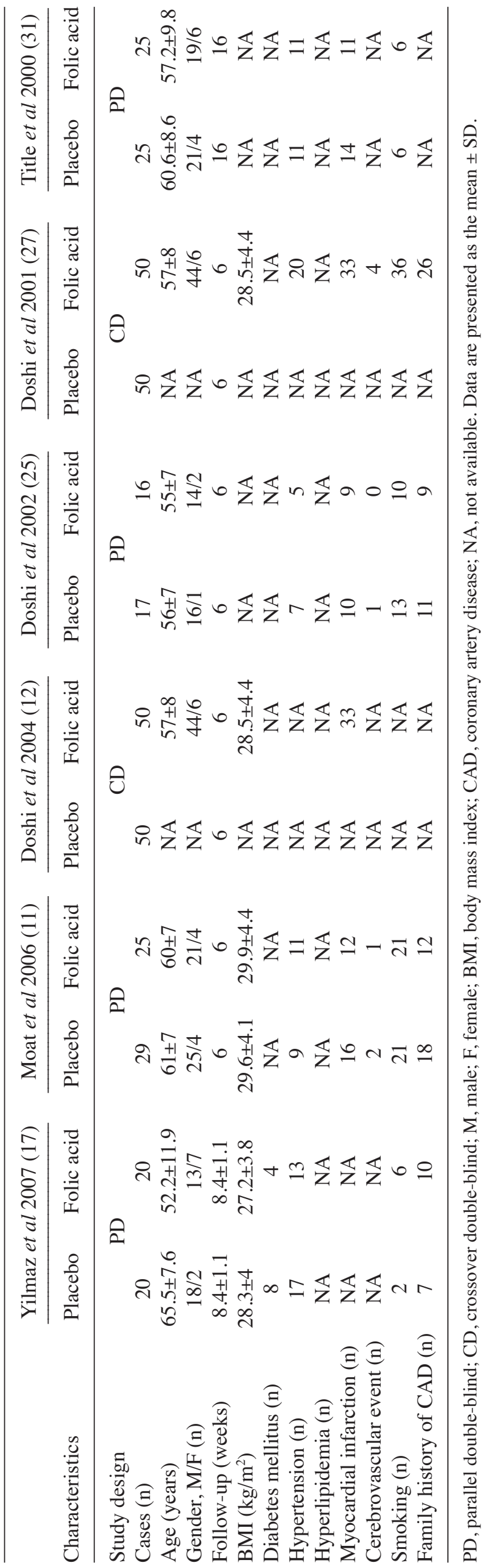

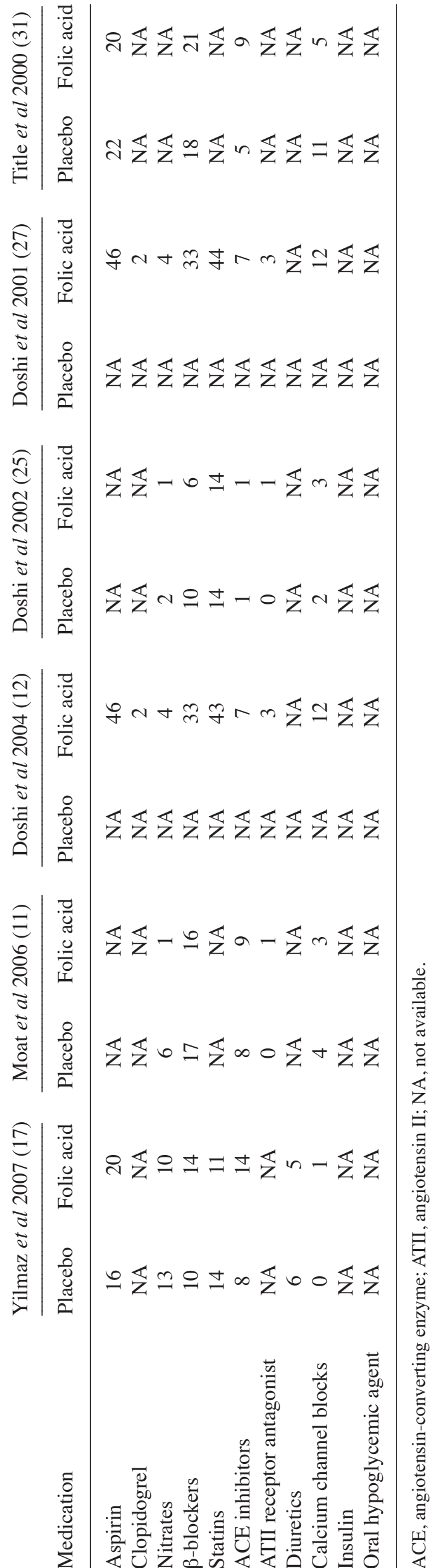




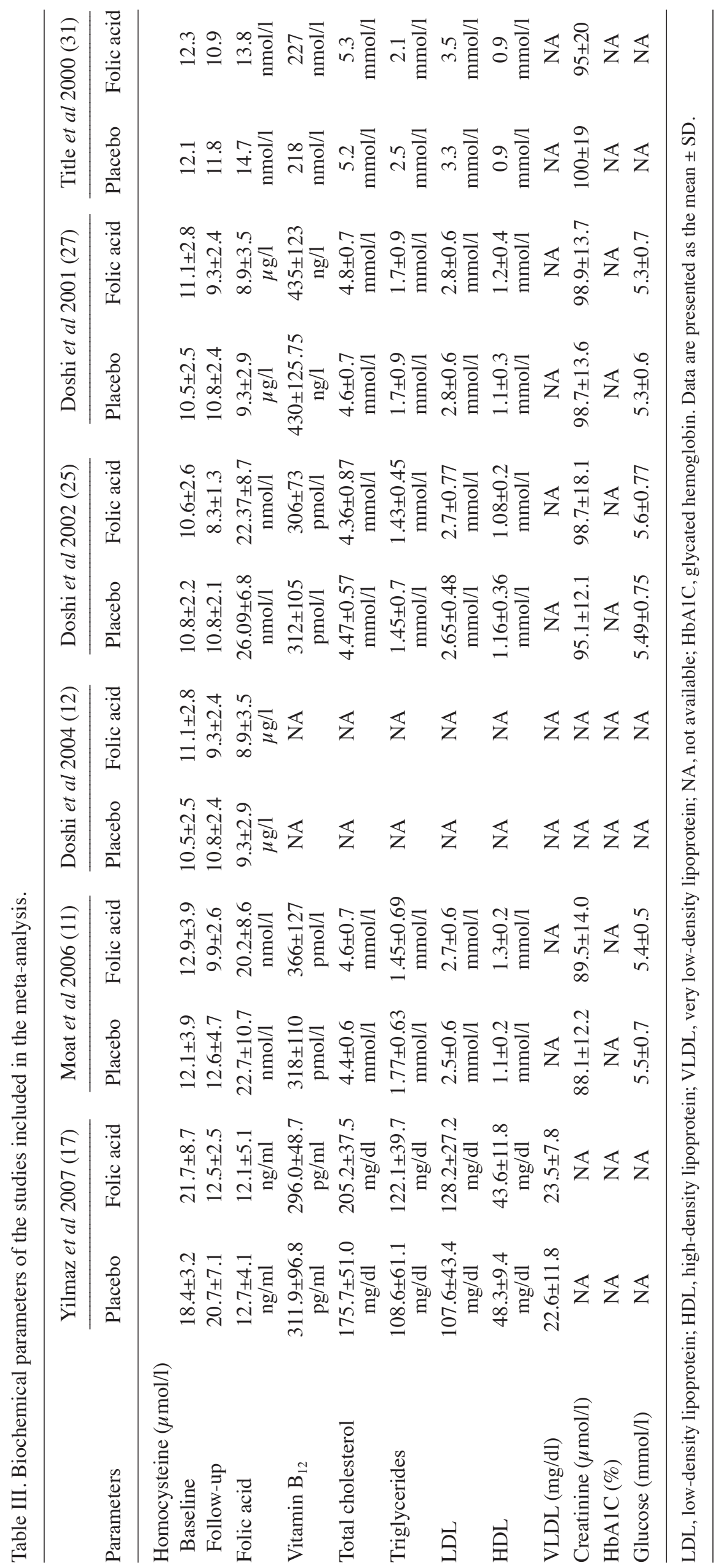


bீ:

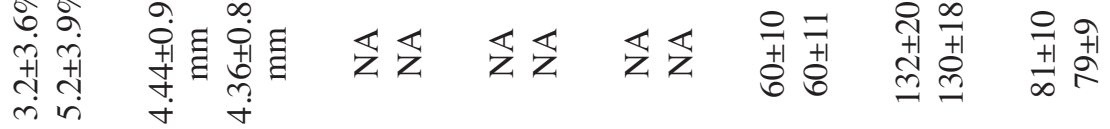

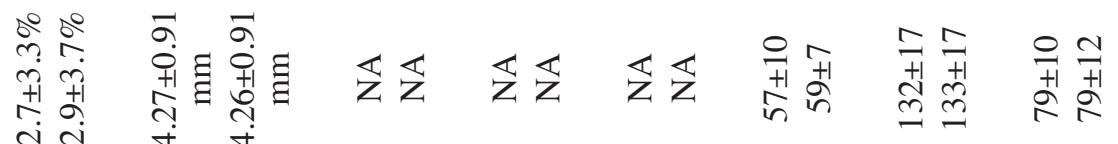

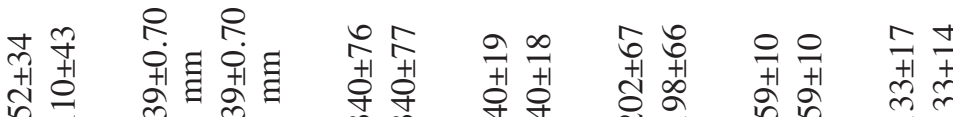

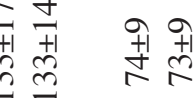

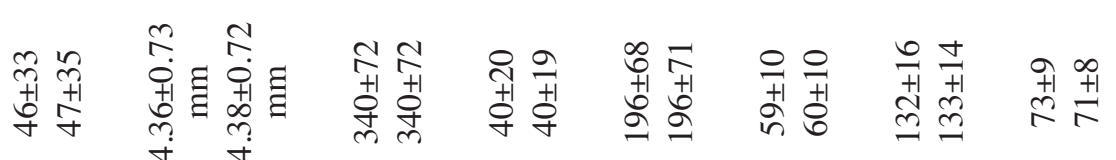

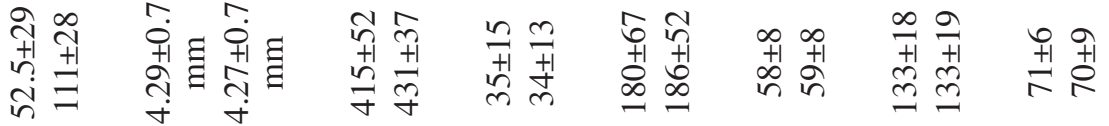

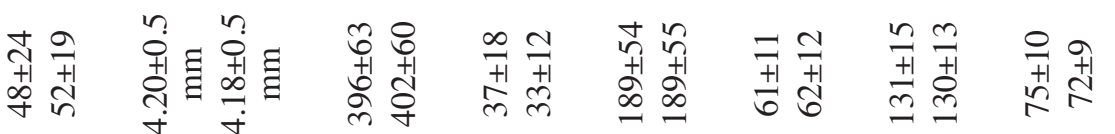

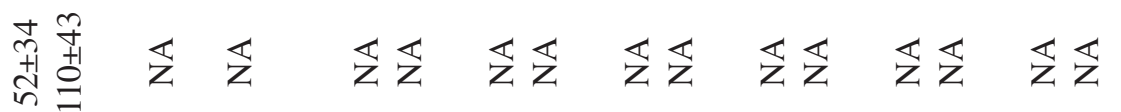

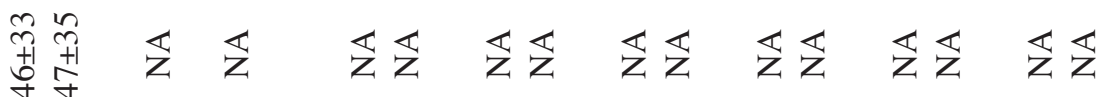

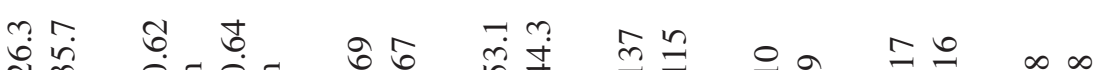

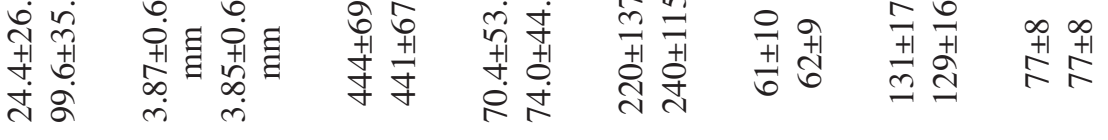

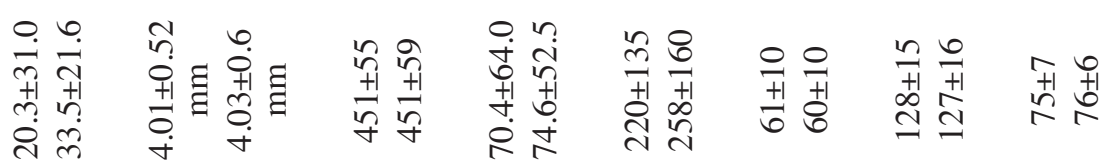

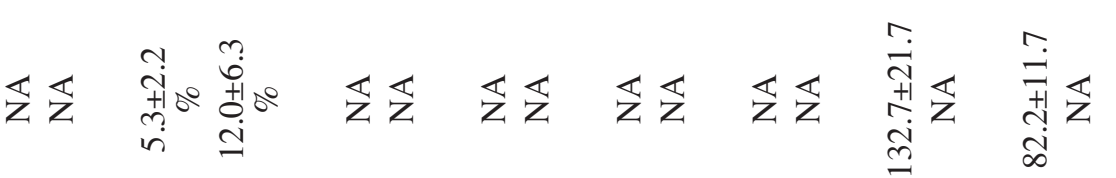

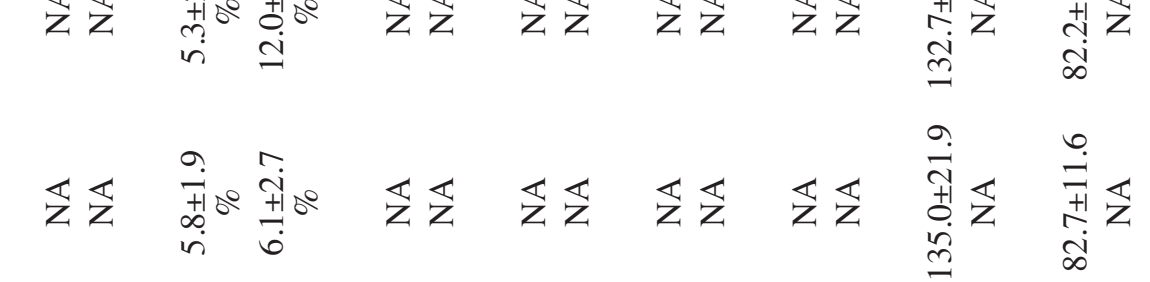

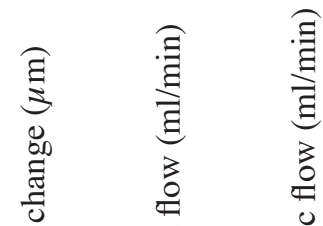




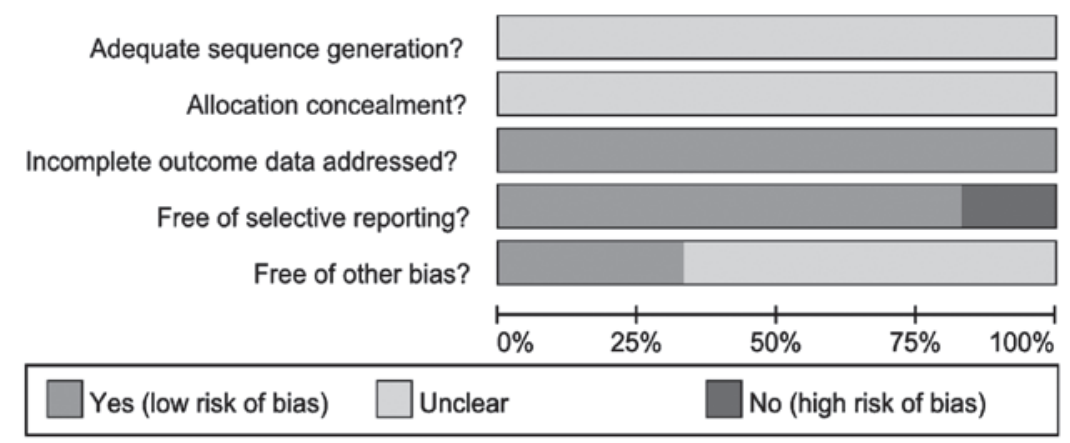

Figure 2. Risk of bias graph. Light grey squares indicate an unclear risk of bias, dark grey squares indicate high risk of bias and medium grey squares indicate low risk of bias.

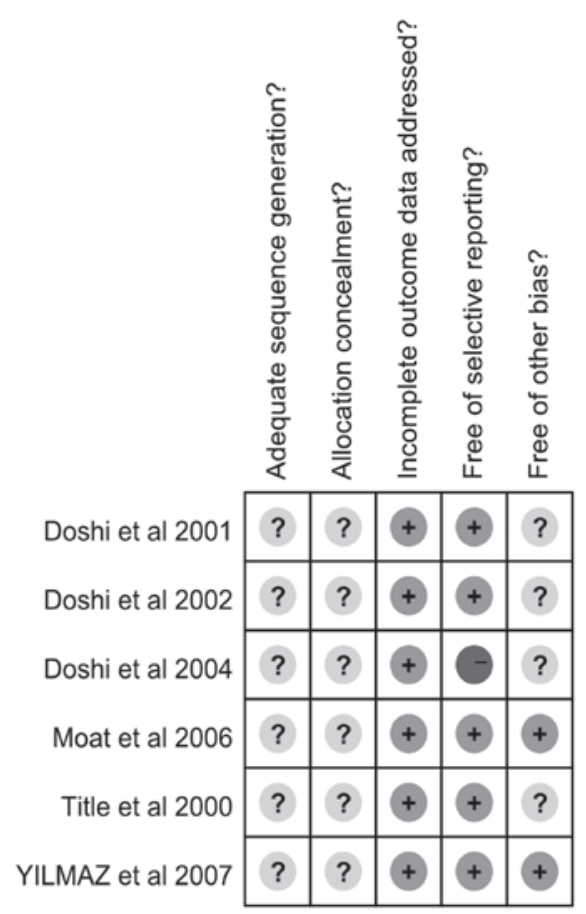

Figure 3. Risk of bias summary. '?' Indicates unclear risk of bias, '-' indicates high risk of bias and ' + ' indicates a low risk of bias.

treatment. The phenomenon, which may be mediated by an increase in nitric oxide (NO) bioavailability and the generation of superoxide ions, was also confirmed by in vitro laboratory experiments $(11,35)$. Schwammenthal et al $(36)$, by performing a meta-analysis of a large number of folic acid clinical trials, predicted that folic acid supplementation at dose of $200 \mu \mathrm{g} /$ day was capable of reducing plasma homocysteine levels by an average of $4 \mu \mathrm{mol} / 1$. In addition, the authors hypothesized that it may be possible to reduce the number of patients succumbing to cardiovascular disease by 13,500-50,000 each year in the USA. A recent meta-analysis of 12 RCTs using 16,958 subjects found that folic acid supplementation had no efficacy on reducing the risk of CAD (37). However, it should be noted that half of the trials included in this meta-analysis used folic acid dosages that were $<5,000 \mu \mathrm{g} / \mathrm{day}$. An additional meta-analysis (38) observed that the changes in BP and FMD, along with the concomitant changes in the risk of coronary heart disease, may only be observed when folic acid doses are in the order of 5,000 $\mu \mathrm{g} /$ day or greater.
The mechanism by which folic acid improves endothelial function remains unclear, however, previous studies have shown that the phenomenon is likely to be associated with the following mechanisms. Firstly, reduced plasma homocysteine levels. Homocysteine is capable of promoting the generation of hydrogen peroxide and oxygen-derived free radicals by the autoxidation of the sulfhydryl on homocysteine, causing the vascular endothelium to be damaged. This results in abnormal changes to the vascular endothelial cell cytoskeleton, accelerating the oxidation of LDL, increasing the formation of foam cells, thickening the walls of blood vessels and even leading to occlusion of blood vessels. Furthermore, homocysteine may also induce apoptosis in endothelial cells and affect the expression of adhesion factors and cytokines, reducing NO-dependent vasorelaxation (39). However, as mentioned previously, high-dose folic acid supplementation improves endothelial function and reduces plasma homocysteine levels, but does not correlate positively (25). A second potential mechanism is that vascular endothelial cells are weakened by oxidative stress. The biological activity of NO directly affects endothelial function and $\mathrm{NO}$ biological activity is determined by the activity of nitric oxide synthase (NOS) and NO inactivation. Various pathophysiological factors are capable of causing the decoupling of eNOS, the result of which produces NO which is converted into generating oxygen-derived free radicals. In recent years, studies have found that the NOS cofactor, tetrahydrobiopterin, is an important regulator of NOS function, which maintains the enzymatic coupling of L-arginine oxidation in order to produce NO (40). NO inactivation is mostly determined by a variety of reactive oxygen-derived free radicals. Thirdly, an additional mechanism may be that folic acid, as a specific type of one-carbon substitution, may be important in repairing genetic damage and maintaining genetic stability (41). Finally, NO production may be directly improved by enhancing the enzymatic activity of eNOS, however, the scavenging potency is 20 -fold lower than that of vitamin C $(42,43)$.

Imbalance in the secretion and release of vasoactive substances due to vascular endothelial cell injury leads to spasming of the coronary artery, rupturing of the coronary artery plaque, platelet aggregation and thrombus formation. In addition, it reduces the antithrombotic ability of endothelial cells and increases blood coagulation, which causes thrombosis, ultimately promoting the occurrence and development of CAD. Therefore, improving endothelial function has great clinical 


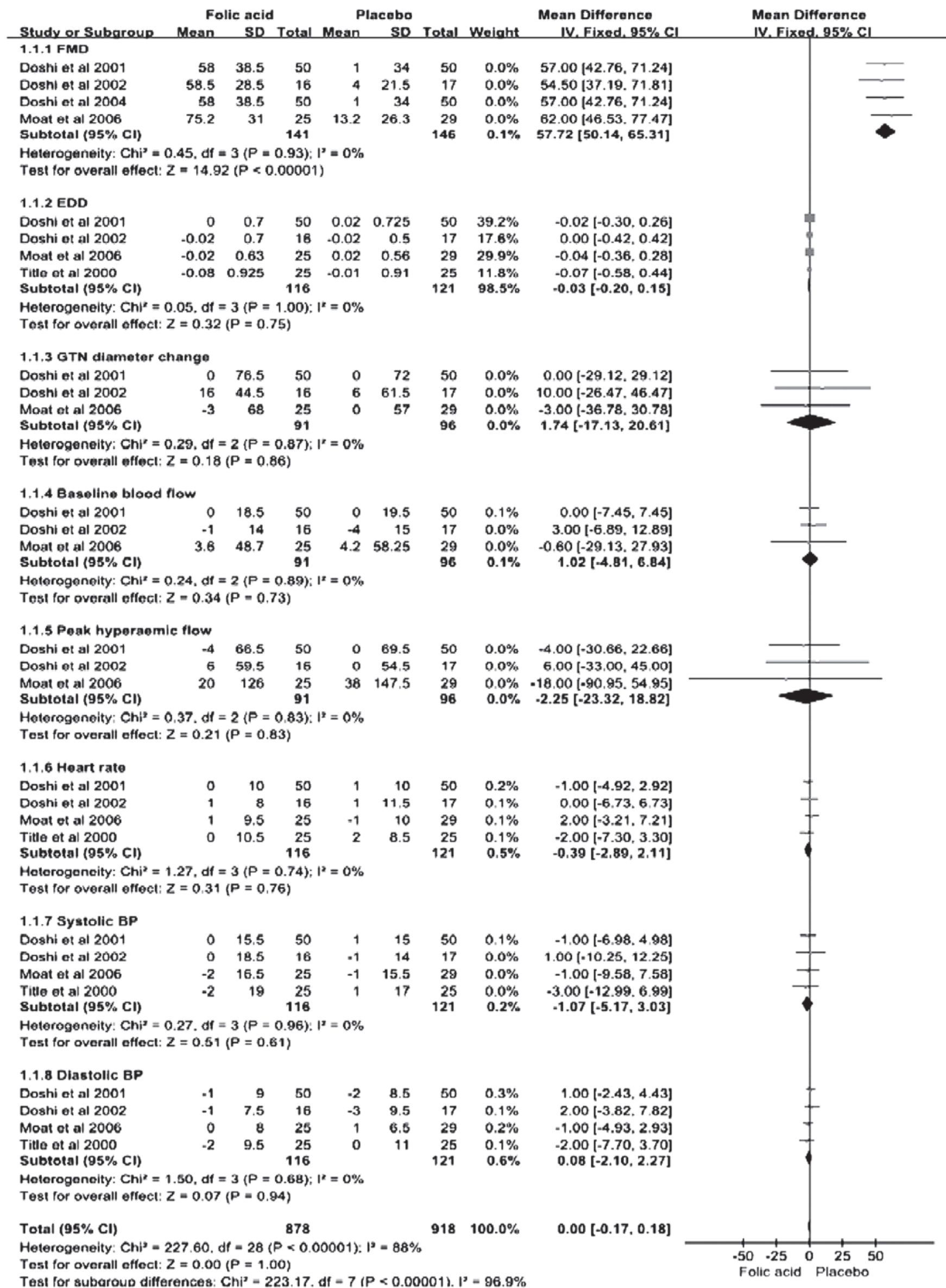

Figure 4. MD with 95\% CI estimates for FMD, EDD, GTN diameter change, heart rate, baseline and peak hyperemic flow, systolic and diastolic BP (folic acid vs. placebo), by summarizing different results of included trials in this study. MD, mean difference; CI, confidence interval; FMD, flow-mediated dilation; EDD, end diastolic diameter; GTN, glyceryl-trinitrate; BP, blood pressure. 


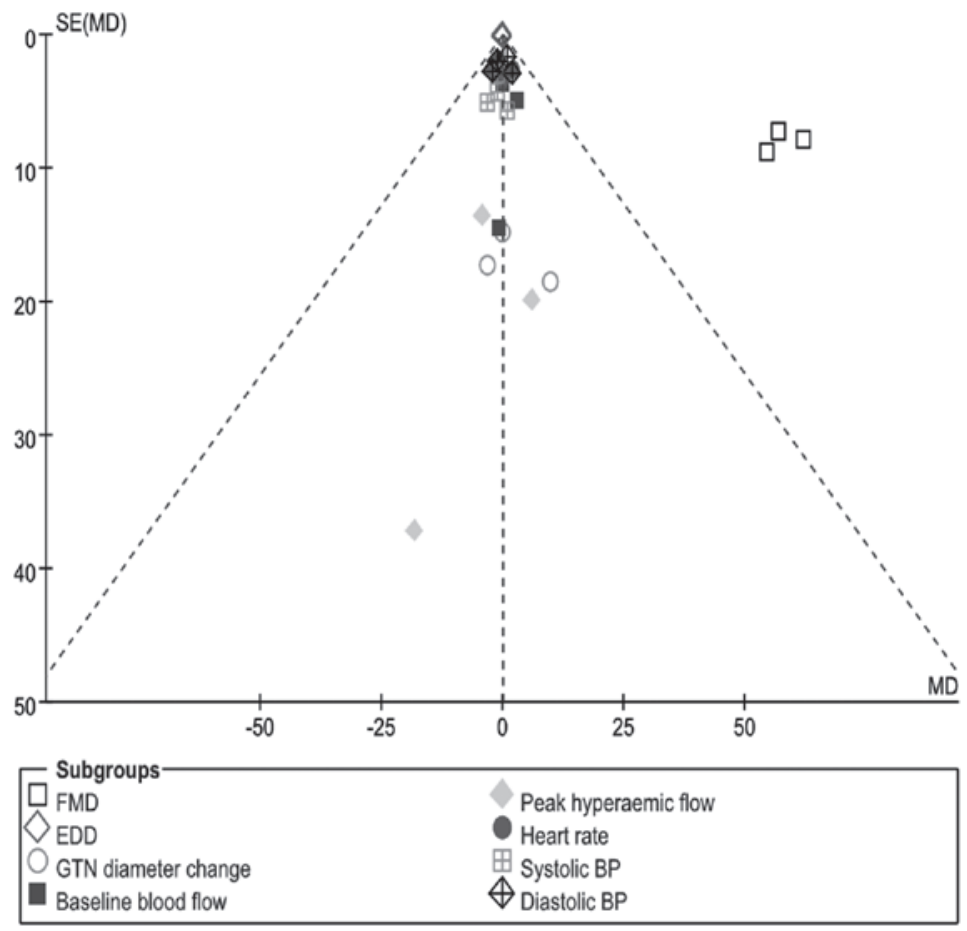

Figure 5. Funnel plot of FMD, EDD, GTN diameter change, heart rate, baseline and peak hyperemic flow, systolic and diastolic BP (folic acid vs. placebo), by summarizing different results of included trials in this study. FMD, flow-mediated dilation; EDD, end diastolic diameter; GTN, glyceryl-trinitrate; BP, blood pressure.

\begin{tabular}{|c|c|c|c|c|c|c|c|c|c|}
\hline \multirow[b]{2}{*}{ Study or Subgroup } & \multicolumn{3}{|c|}{ Folic acid } & \multicolumn{3}{|c|}{ Placebo } & & \multirow{2}{*}{$\begin{array}{l}\text { Mean Difference } \\
\text { IV, Random, } 95 \% \mathrm{Cl}\end{array}$} & \multirow{2}{*}{$\begin{array}{c}\text { Mean Difference } \\
\mid V \text {, Random, } 95 \% \mathrm{Cl}\end{array}$} \\
\hline & Mean & SD & Total & Mean & SD & Total & Weight & & \\
\hline Doshi et al 2001 & -1.8 & 2.6 & 50 & 0.3 & 2.45 & 50 & $23.1 \%$ & $-2.10[-3.09,-1.11]$ & - \\
\hline Doshi et al 2002 & -2.3 & 1.95 & 16 & 0 & 2.15 & 17 & $21.6 \%$ & $-2.30[-3.70,-0.90]$ & - \\
\hline Doshi et al 2004 & -1.8 & 2.6 & 50 & 0.3 & 2.45 & 50 & $23.1 \%$ & $-2.10[-3.09,-1.11]$ & - \\
\hline Moat et al 2006 & -3 & 3.25 & 25 & 0.5 & 4.3 & 29 & $18.9 \%$ & $-3.50[-5.52,-1.48]$ & - \\
\hline YILMAZ et al 2007 & -9.2 & 5.6 & 20 & 2.3 & 5.15 & 20 & $13.3 \%$ & $-11.50[-14.83,-8.17]$ & $\rightarrow$ \\
\hline Total $(95 \% \mathrm{Cl})$ & & & 161 & & & 166 & $100.0 \%$ & $-3.66[-5.44,-1.87]$ & 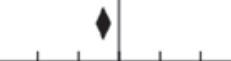 \\
\hline \multicolumn{9}{|c|}{$\begin{array}{l}\text { Heterogeneity: } \operatorname{Tau}^{2}=3.33 ; \mathrm{Ch}^{2}=30.24, \mathrm{df}=4(\mathrm{P}<0.00001) ; \mathrm{I}^{2}=87 \% \\
\text { Test for overall effect: } Z=4.02(P<0.0001)\end{array}$} & 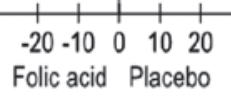 \\
\hline
\end{tabular}

Figure 6. MD with 95\% CI estimates for plasma homocysteine concentration (folic acid vs. placebo), by summarizing different results of included trials in this study. MD, mean difference; CI, confidence interval.

value for the prevention and treatment of CAD. The present meta-analysis predicts that the long-term use of high-dose folic acid may reduce the concentration of plasma homocysteine and increase FMD, improving endothelial function. Subsequently, the prevention and treatment of CAD may be achieved via clinical trials of folic acid intervention with CAD patients.

It is possible that high-doses of folic acid (5 mg daily) administered orally improves endothelial function and lowers the concentration of plasma homocysteine in CAD patients. Folic acid supplementation is inexpensive, potentially effective and temporarily devoid of adverse effects. Therefore, folic acid has an exceptionally favorable benefit/risk ratio for improving endothelial function in CAD patients.

There are several limitations to consider when interpreting the results of the present study. Firstly, only two trials included in the meta-analysis truly divided the patients into folic acid supplementation and placebo groups. In the additional fours studies, the patients were randomized to folic acid $5 \mathrm{mg}$ or folic acid $400 \mu \mathrm{g} / \mathrm{N}$-acetylcysteine/folic acid combined with vitamin $\mathrm{B}$ or a placebo. An additional limitation was the small number of cases. No report documented data on the side effects of high dose folic acid and the studies also had markedly different durations and evaluation indices for endothelial function. Thirdly, the current study is prone to the well-known limitation of meta-analyses, namely variation in study design and publication bias. Furthermore, the meta-analytical approach with observational data is even more fraught with limitations. Thus, additional double-blind, randomized, placebo-controlled, multicenter studies with high quality and longer follow-up periods are required to confirm the conclusions of the present study. It will be useful to observe whether the efficacy of folic acid supplementation, particularly on arterial function, is similar among patients with angina, myocardial infarction and non symptom coronary heart disease. 
In conclusion, the present meta-analysis of RCTs demonstrates that folic acid supplementation of $5 \mathrm{mg} /$ day for $>4$ weeks significantly improves FMD and lowers plasma homocysteine concentration in patients with CAD. Thus, this study has underlined the importance of high-dose folic acid supplementation for the improvement of endothelial function. Folic acid supplementation should be recommended for CAD patients. However, more RCTs are required in order to confirm this meta-analysis.

\section{Acknowledgements}

The authors thank all the members of the Department of Cardiology and Cardiovascular Research Institute of Renmin Hospital of Wuhan University for their assistance and supportive advice. The study was supported by a grant from the National Natural Science Foundation of China (no. 81170307).

\section{References}

1. Quyyumi AA: Prognostic value of endothelial function. Am J Cardiol 91: 19H-24H, 2003.

2. Weber T, Auer J, O'Rourke MF, et al: Arterial stiffness, wave reflections, and the risk of coronary artery disease. Circulation 109: 184-189, 2004.

3. Gupta SK, Kotwal J, Kotwal A, Dhall A and Garg S: Role of homocysteine \& MTHFR C677T gene polymorphism as risk factors for coronary artery disease in young Indians. Indian J Med Res 135: 506-512, 2012.

4. Vinukonda G, Shaik Mohammad N, Md Nurul Jain J, Prasad Chintakindi K and Rama Devi Akella R: Genetic and environmental influences on total plasma homocysteine and coronary artery disease (CAD) risk among South Indians. Clin Chim Acta 405: 127-131, 2009.

5. Lin PT, Huang MC, Lee BJ, Cheng CH, Tsai TP and Huang YC: High plasma homocysteine is associated with the risk of coronary artery disease independent of methylenetetrahydrofolate reductase $677 \mathrm{C}-->\mathrm{T}$ genotypes. Asia Pac J Clin Nutr 17: 330-338, 2008

6. Bleie Ø, Strand E, Ueland PM, Vollset SE, Refsum H, Igland J, Nordrehaug JE and Nygård OK: Coronary blood flow in patients with stable coronary artery disease treated long term with folic acid and vitamin B12. Coron Artery Dis 22: 270-278, 2011.

7. MacKenzie KE, Wiltshire EJ, Gent R, Hirte C, Piotto L and Couper JJ: Folate and vitamin B6 rapidly normalize endothelial dysfunction in children with type 1 diabetes mellitus. Pediatrics 118: 242-253, 2006.

8. Till U, Röhl P, Jentsch A, et al: Decrease of carotid intima-media thickness in patients at risk to cerebral ischemia after supplementation with folic acid, vitamins B6 and B12. Atherosclerosis 181: 131-135, 2005.

9. de Bree A, van Mierlo LA and Draijer R: Folic acid improves vascular reactivity in humans: a meta-analysis of randomized controlled trials. Am J Clin Nutr 86: 610-617, 2007.

10. Shirodaria C, Antoniades C, Lee J, et al: Global improvement of vascular function and redox state with low-dose folic acid: implications for folate therapy in patients with coronary artery disease. Circulation 115: 2262-2270, 2007.

11. Moat SJ, Madhavan A, Taylor SY, et al: High- but not low-dose folic acid improves endothelial function in coronary artery disease. Eur J Clin Invest 36: 850-859, 2006.

12. Doshi SN, Moat SJ, Lewis MJ, McDowell IF, Giddings JC and Goodfellow J: Short-term high-dose folic acid does not alter markers of endothelial cell damage in patients with coronary heart disease. Int J Cardiol 94: 203-207, 2004.

13. Guo H, Chi J, Xing Y and Wang P: Influence of folic acid on plasma homocysteine levels \& arterial endothelial function in patients with unstable angina. Indian J Med Res 129: 279-284, 2009.

14. Tam WY, Chook P, Qiao M, et al: The efficacy and tolerability of adjunctive alternative herbal medicine (Salvia miltiorrhiza and Pueraria lobata) on vascular function and structure in coronary patients. J Altern Complement Med 15: 415-421, 2009.
15. Myles PS, Leslie K, Peyton P, et al: Nitrous oxide and perioperative cardiac morbidity (ENIGMA-II) trial: rationale and design. Am Heart J 157: 488-494, 2009.

16. Sultan N, Khan MA and Malik S: Effect of folic acid supplementation on homocysteine level in postmenopausal women. J Ayub Med Coll Abbottabad 19: 78-81, 2007.

17. Yilmaz H, Sahin S, Sayar N, et al: Effects of folic acid and $\mathrm{N}$-acetylcysteine on plasma homocysteine levels and endothelial function in patients with coronary artery disease. Acta Cardiol 62: 579-585, 2007.

18. Austen SK, Fassett RG, Geraghty DP and Coombes JS: Folate supplementation fails to affect vascular function and carotid artery intima media thickness in cyclosporin A-treated renal transplant recipients. Clin Nephrol 66: 373-379, 2006.

19. Fernández-Miranda $\mathrm{C}$, Yebra M, Aranda JL, Gómez $\mathrm{P}$, Martínez J, Núñez V and Gómez de la Cámara A: Effect of folic acid treatment on carotid intima-media thickness of patients with coronary disease. Int J Cardiol 118: 345-349, 2007.

20. Chia S, Wilson R, Ludlam CA, Webb DJ, Flapan AD and Newby DE: Endothelial dysfunction in patients with recent myocardial infarction and hyperhomocysteinaemia: effects of vitamin supplementation. Clin Sci (Lond) 108: 65-72, 2005.

21. Doshi S, McDowell I, Moat S, Lewis M and Goodfellow J: Folate improves endothelial function in patients with coronary heart disease. Clin Chem Lab Med 41: 1505-1512, 2003.

22. Woodman RJ, Celermajer DE, Thompson PL and Hung J: Folic acid does not improve endothelial function in healthy hyperhomocysteinaemic subjects. Clin Sci (Lond) 106: 353-358, 2004

23. Liem A, Reynierse-Buitenwerf GH, Zwinderman AH, Jukema JW and van Veldhuisen DJ: Secondary prevention with folic acid: effects on clinical outcomes. J Am Coll Cardiol 41: 2105-2113, 2003.

24. Willems FF, Aengevaeren WR, Boers GH, Blom HJ and Verheugt FW: Coronary endothelial function in hyperhomocysteinemia: improvement after treatment with folic acid and cobalamin in patients with coronary artery disease. J Am Coll Cardiol 40: 766-772, 2002.

25. Doshi SN, McDowell IF, Moat SJ, Payne N, Durrant HJ, Lewis MJ and Goodfellow J: Folic acid improves endothelial function in coronary artery disease via mechanisms largely independent of homocysteine lowering. Circulation 105: 22-26, 2002.

26. Miner SE, Cole DE, Evrovski J, Forrest Q, Hutchison S, Holmes K and Ross HJ: Pyridoxine improves endothelial function in cardiac transplant recipients. J Heart Lung Transplant 20: 964-969, 2001.

27. Doshi SN, McDowell IF, Moat SJ, Lang D, Newcombe RG, Kredan MB, Lewis MJ and Goodfellow J: Folate improves endothelial function in coronary artery disease: an effect mediated by reduction of intracellular superoxide? Arterioscler Thromb Vasc Biol 21: 1196-1202, 2001.

28. Thambyrajah J, Landray MJ, Jones HJ, McGlynn FJ, Wheeler DC and Townend JN: A randomized double-blind placebo-controlled trial of the effect of homocysteine-lowering therapy with folic acid on endothelial function in patients with coronary artery disease. J Am Coll Cardiol 37: 1858-1863, 2001.

29. Spence JD, Howard VJ, Chambless LE, Malinow MR, Pettigrew LC, Stampfer M and Toole JF: Vitamin Intervention for Stroke Prevention (VISP) trial: rationale and design. Neuroepidemiology 20: 16-25, 2001

30. Chambers JC, Ueland PM, Obeid OA, Wrigley J, Refsum H and Kooner JS: Improved vascular endothelial function after oral B vitamins: An effect mediated through reduced concentrations of free plasma homocysteine. Circulation 102: 2479-2483, 2000.

31. Title LM, Cummings PM, Giddens K, Genest JJ Jr and Nassar BA: Effect of folic acid and antioxidant vitamins on endothelial dysfunction in patients with coronary artery disease. J Am Coll Cardiol 36: 758-765, 2000.

32. Moens AL, Goovaerts I, Claeys MJ and Vrints CJ: Flow-mediated vasodilation: a diagnostic instrument, or an experimental tool? Chest 127: 2254-2263, 2005.

33. Yoshida T, Kawano H, Miyamoto S, Motoyama T, Fukushima H, Hirai N and Ogawa H: Prognostic value of flow-mediated dilation of the brachial artery in patients with cardiovascular disease. Intern Med 45: 575-579, 2006.

34. Fairfield KM and Fletcher RH: Vitamins for chronic disease prevention in adults: scientific review. JAMA 287: 3116-3126, 2002.

35. Verhaar MC, Wever RM, Kastelein JJ, van Dam T, Koomans HA and Rabelink TJ: 5-methyltetrahydrofolate, the active form of folic acid, restores endothelial function in familial hypercholesterolemia. Circulation 97: 237-241, 1998. 
36. Schwammenthal Y and Tanne D: Homocysteine, B-vitamin supplementation, and stroke prevention: from observational to interventional trials. Lancet Neurol 3: 493-495, 2004.

37. Bazzano LA, Reynolds K, Holder KN and He J: Effect of folic acid supplementation on risk of cardiovascular diseases: a meta-analysis of randomized controlled trials. JAMA 296: 2720-2726, 2006

38. McRae MP: High-dose folic acid supplementation effects on endothelial function and blood pressure in hypertensive patients: a meta-analysis of randomized controlled clinical trials. J Chiropr Med 8: 15-24, 2009.

39. Dionisio N, Jardín I, Salido GM and Rosado JA: Homocysteine, intracellular signaling and thrombotic disorders. Curr Med Chem 17: 3109-3119, 2010.
40. Crabtree MJ and Channon KM: Synthesis and recycling of tetrahydrobiopterin in endothelial function and vascular disease. Nitric Oxide 25: 81-88, 2011.

41. Smulders YM and Stehouwer CD: Folate metabolism and cardiovascular disease. Semin Vasc Med 5: 87-97, 2005.

42. Stroes ES, van Faassen EE, Yo M, Martasek P, Boer P, Govers R and Rabelink TJ: Folic acid reverts dysfunction of endothelial nitric oxide synthase. Circ Res 86: 1129-1134, 2000.

43. Hyndman ME, Verma S, Rosenfeld RJ, Anderson TJ and Parsons HG: Interaction of 5-methyltetrahydrofolate and tetrahydrobiopterin on endothelial function. Am J Physiol Heart Circ Physiol 282: H2167-H2172, 2002. 